REZENSIONEN

\section{Nachhaltigkeit auf dem Prüfstand}

\author{
A. Grunwald, J. Kopfmüller: Nachhaltig- \\ keit. Frankfurt a. M., New York: Campus \\ Verlag, 2006, 189 S., ISBN 978-3-593- \\ 37978-4, $€ 12,90$
}

\section{J. Kopfmüller (Hg.): Ein Konzept auf dem Prüfstand. Das integrative Nach- haltigkeitskonzept in der Forschungs- praxis. Berlin: edition sigma, 2006, 330 S., ISBN 978-3-894-04582-1, € 22,90}

\section{Rezension von Achim Daschkeit, Umweltbundesamt}

Es gibt mit Sicherheit Menschen, die das Themenfeld Nachhaltigkeit ,nicht mehr hören können“. Immerhin wird in der Gesellschaft mindestens seit dem Rio-Gipfel (1992), eher noch seit dem Brundtland-Report (1987) intensiv hierüber geredet und geschrieben. Und in der Tat: Der Nachhaltigkeitsdiskurs wird in Wissenschaft, Politik, Verwaltung, Umweltund Unternehmensverbänden, in ,der“ Zivilgesellschaft und an vielen anderen Stellen geführt: Unternehmen fertigen Nachhaltigkeitsberichte an, es bestehen nach wie vor noch eine Vielzahl von Lokale-Agenda21-Prozessen, seit einigen Jahren gibt es hierzulande einen Rat für Nachhaltige Entwicklung usw. Außerdem wird dieser Diskurs sowohl auf internationaler, europäischer, nationaler und kommunaler Ebene geführt. Angesichts der damit verbundenen Produktion von Publikationen und anderweitigen Dokumenten sowie Aktivitäten, die hieraus resultieren, ist man geneigt $\mathrm{zu}$ kapitulieren, wenn man sich einen Überblick verschaffen möchte. Gleichzeitig berührt der Nachhaltigkeitsdiskurs fast alle Lebensbereiche, mit anderen Worten: Man kommt kaum darum herum, sich irgendwann einmal mit der Nachhaltigkeitsthematik auseinanderzusetzen.

\section{Einführung in die Nachhaltigkeit}

Genau in dem Moment ist man gut beraten, das Buch „Nachhaltigkeit“" von Armin Grunwald und Jürgen Kopfmüller zurate zu ziehen, das im Campus Verlag in der Reihe „Einführungen“" erschienen ist. Man erfährt dort $u$. a. etwas zur Entstehungsgeschichte und zu den grundlegenden Prämissen des Konzeptes Nachhaltiger Entwicklung. Nachhaltige Entwicklung wird konkretisiert im Hinblick auf die Messbarkeit mit Indikatoren; es werden gesellschaftliche Handlungsfelder, gesellschaftliche Akteure sowie politische Umsetzungsebenen beschrieben. Weiterhin geht es in dieser Einführung um verschiedene Konzeptionen nachhaltiger Entwicklung - als Stichworte seien hier nur das Säulenkonzept sowie der integrative Ansatz der Helmholtz-Gemeinschaft (HGF) genannt (dazu später mehr). Letztlich werden die Rezeptionsgeschichte betrachtet sowie kritische Aspekte des Nachhaltigkeitskonzeptes beleuchtet.

Um es gleich zu sagen: Es ist erstaunlich, wie die beiden Autoren es schaffen, angesichts des knappen Raumes eines Einführungsbuches viele (wenn auch natürlich nicht alle) Facetten des Nachhaltigkeitsdiskurses anzusprechen und so aufzubereiten, dass der Leser interessiert ist, sich weiter mit dem Thema zu beschäftigen. Exemplarisch soll nur auf einige inhaltliche Aspekte hingewiesen werden:

- In historischer Betrachtung betonen die Autoren, dass die allgemeine Zustimmung zum Nachhaltigkeitskonzept - als Vorbedingung zu dessen weiterer Verbreitung auch deswegen so „einfach“ gewesen sei, weil die Formulierungen im BrundtlandBericht von 1987 so allgemein gehalten und wenig konkret seien, dass für jeden Akteur hinreichende Interpretationsspielräume bestanden (und zum Teil noch bestehen). Man konnte sich also auf die Idee von Nachhaltigkeit einlassen, ohne tief greifende Veränderungen in seinem eigenen Handlungsfeld befürchten zu müssen. Am Beispiel von Unternehmen erläutern Grunwald und Kopfmüller, dass eine substanzielle Orientierung an Nachhaltigkeitskriterien nur in wenigen Unternehmen $\mathrm{zu}$ beobachten sei, und $\mathrm{zu}-$ meist nur dann, wenn „Kosteneinsparungen und Gewinnsteigerungen" absehbar seien. 
Ansonsten fände nachhaltigkeitsorientiertes Verhalten oftmals nicht statt (S. 110).

- Die Leser erfahren auch die ernüchternde Feststellung, dass nach gut zwanzig Jahren Nachhaltigkeitsdiskussion bislang immer noch recht wenig indikatorenbasierte Umsetzungen vorliegen; hierdurch ließe sich aber relativ konkret nachzeichnen, ob die Nachhaltigkeitsdiskussion eine nachhaltige (sic!) gesellschaftliche Wirkung hatte. Die Autoren schlagen vor, Nachhaltigkeit eher im Sinne einer regulativen Idee zu verstehen denn als „hartes“ Instrument gesellschaftlicher Steuerung. Ich persönlich sehe hierin die Gefahr, dass die Nachhaltigkeitsdiskussion dadurch wieder (noch) allgemeiner und weniger verbindlich werden könnte.

- Vor diesem Hintergrund ist das Kapitel 5 (Nachhaltige Entwicklung konkret, S. 59ff.) meines Erachtens von zentraler Bedeutung in dieser Einführung. In diesem Kapitel erfolgen einige Hinweise auf IndikatorenAnsätze, die natürlich aus Platzgründen in der Einführung nicht im Detail ausgeführt werden können. Es ist in diesem Zusammenhang auch legitim, wenn die Autoren auf den integrativen Nachhaltigkeitsansatz der HGF hinweisen (die Autoren sind hieran maßgeblich beteiligt). Der HGF-Ansatz schlägt, theoretisch fundiert, Indikatoren zur Messung von Nachhaltigkeit vor, die sich eben nicht an dem klassischen Säulenkonzept (Ökonomie, Ökologie, Soziales, Institutionen) orientieren.

\section{Das integrative HGF-Nachhaltigkeits- konzept}

Genau dieser letzte Punkt führt zum zweiten Buch, auf das hier hingewiesen wird. Herausgeber ist - wie ersichtlich - einer der Autoren der „Einführung“ in die Nachhaltigkeit. Im Mittelpunkt des von Jürgen Kopfmüller herausgegebenen Bandes „Ein Konzept auf dem Prüfstand. Das integrative Nachhaltigkeitskonzept in der Forschungspraxis" steht das bereits erwähnte integrative Nachhaltigkeitskonzept der HGF, das seit ca. sieben Jahren entwickelt, erprobt und auch in der Praxis umgesetzt wird. In diesem Sammelband geht es vorrangig um die Forschungspraxis derjenigen, die mit diesem integrativen HGF-Ansatz arbeiten. Damit geht es nicht in erster Linie um die praktische Umsetzung von Nachhaltigkeit.

Der Sammelband ist in drei Blöcke unterteilt: Im ersten Block geht es um das Konzept selbst, die Kapitel beinhalten einige reflektierende Beiträge. Im zweiten Block werden Anwendungen des Konzepts geschildert. Nach eigenen Aussagen geht es um einen „Blumenstrau $\beta^{\text {“ }}$ ganz verschiedener Themen: Die Beiträge reichen von Nachhaltigkeitskriterien für Nachwachsende Rohstoffe über die Verwendbarkeit des HGF-Ansatzes zur Bewertung börsennotierter Unternehmen bis hin Anwendungen im Bereich Megacities. Im dritten Block geht es um geplante bzw. prinzipiell mögliche Anwendungen. Basis des Buches ist ein sogenannter Anwender-Workshop, der im November 2004 stattgefunden hat - mittlerweile dürfte also die Entwicklung deutlich weiter vorangeschritten sein. Ziel des Workshops war u. a. die Netzwerkbildung unter denjenigen, die mit dem HGF-Ansatz arbeiten.

Für den Block „Anwendungen“ ergibt sich ein grundsätzliches Dilemma: Es ist verständlich, wenn jeder das HGF-Konzept auf seine Fragestellung (eine bestimmte Region, ein bestimmtes Thema, eine bestimmte Branche usw.) zuschneidert. Je mehr diese spezifischen Konkretisierungen erfolgen, desto schwieriger wird es naturgemäß, verallgemeinernde Aussagen zu treffen, eben weil sich die Konkretisierungen dann doch wesentlich voneinander unterscheiden. Dennoch ist dieser Teil „Anwendungen“ sehr aufschlussreich, nicht zuletzt deswegen, weil der Herausgeber Jürgen Kopfmüller einen im wahrsten Sinne des Wortes „Rahmen setzenden" Überblicksbeitrag an den Beginn des Buches gesetzt hat. Dieser Beitrag ist deswegen hervorhebenswert, weil er die Randbedingungen des HGF-Nachhaltigkeitsansatzes noch einmal aufgreift und dabei Defizite und Verbesserungsmöglichkeiten keineswegs verschweigt.

Außerdem geht Kopfmüller auf „Facetten der Integration" ein - ein wahrlich zentrales Thema im integrativen HGF-Ansatz:

- Zum einen dienen die sogenannten Nachhaltigkeitsregeln als Integrationsmöglichkeit. Kopfmüller verweist darauf, dass diese Regeln abwägungsfähig konzipiert sein müssten. An dieser Stelle hätte ich mir methodische Aussagen erhofft, wie denn nun diese Abwägung konkret vorgenommen 
werden könnte: Ist eine verbal-argumentative Abwägung gemeint oder ein anderes methodisches Vorgehen?

- Ein zweites Integrationselement bestünde „,in der Betrachtung der gesamten Analysekette und der Wechselwirkungen der einzelnen Elemente" (S. 31). Konkret nennt Kopfmüller die Formulierung grundlegender Handlungsorientierungen, deren Konkretisierung durch geeignete Indikatoren, die Festlegung von Zielwerten für diese Indikatoren, die Identifikation und Analyse prioritärer Problemlagen sowie die Entwicklung und Analyse von Handlungsstrategien zur Erreichung gesetzter Ziele bzw. zur Lösung bestehender Probleme.

- Drittens erwähnt der Autor die Möglichkeit der Integration in räumlicher Hinsicht (Interaktionen zwischen Regionen oder zwischen lokaler, nationaler und internationaler Ebene).

Die Beispiele im Block „Anwendungen“ sollen hier nicht im Einzelnen diskutiert werden; dennoch sei auf die teils völlig unterschiedliche Strukturierung bei der Anwendung des integrativen HGF-Konzepts hingewiesen. Der Beitrag „Downscaling von Nachhaltigkeit. Das Integrative Nachhaltigkeitskonzept als Bauplan für kommunale Indikatorensysteme" (Gerhard Hartmuth et al.) beispielsweise ist als Praxisanwendung im kommunalen Bereich anzusehen und zugleich ein ambitionierter Versuch, einen Indikatorenansatz zu entwickeln. Neben dieser Form der Operationalisierung ist das Einbinden des Leitbilds der Nachhaltigen Entwicklung in das lokale Setting gewährleistet (Kontextualisierung). Das Helmholtz-Konzept wurde als „Bauplan" beim Prozess des Herunterbrechens des abstrakten und globalen Nachhaltigkeitskonzeptes und als Folie für die Entwicklung von Nachhaltigkeitsindikatoren verwendet. Dieses „Herunterbrechen" erfolgte in vier Schritten: Analyse lokaler Problemlagen, Übertragen/ Modifizieren der Nachhaltigkeitsregeln, Betrachtung der lokalen Problemlagen im Licht der Nachhaltigkeitsregeln, Auswahl der Nachhaltigkeitsindikatoren.

Auf die Frage, ob ,der Bauplan taugt“, ob also das integrative Nachhaltigkeitskonzept in Form eines Indikatorensystems für die kommunale Praxis umsetzbar ist, antworten die Autoren mit einem klaren Ja. Der Rekurs auf den HGF-Ansatz erweist sich als systematische und konsistente Folie und ist gleichzeitig flexibel genug für lokale Anpassungen.

Ein ähnlich strukturiertes Vorgehen wie bei Hartmuth et al. hätte man sich auch für viele andere konkrete Anwendungsbeispiele gewünscht. Im Beitrag „Die Anwendung des integrativen Konzepts auf das Aktivitätsfeld ,Freizeit und Tourismus in Deutschland'“ (Sigrid Klein-Vielhauer) verbleibt die Diskussion größtenteils auf der konzeptionellen Ebene und der Darstellung „Nachhaltige Entwicklung in Megacities. Die HGF-Forschungsinitiative ,Risk Habitat Megacity'“ (Jürgen Kopfmüller, Helmut Lehn) ist ebenfalls noch sehr abstrakt. Man merkt, dass offenbar ein Forschungsantrag die Basis des Beitrages war. Die beiden zuletzt genannten Beiträge hätten somit eher in den letzten Block „Geplante und mögliche Anwendungen“ gehört. Unabhängig davon: Der Band ist aus meiner Sicht sehr instruktiv, weil er sowohl in konstruktiver als auch in kritisch-reflektierender Hinsicht einen guten Einblick in den Stand der (Weiter-)Entwicklung des HGF-Projektes bietet.

\section{Fazit}

Es ist äußerst sinnvoll, die beiden angezeigten Bücher im Zusammenhang zu lesen. Die „Einführung" zielt eher auf Leser, die sich bislang vielleicht noch nicht so intensiv mit dem Thema Nachhaltigkeit beschäftigt haben. Die Lektüre des Sammelbandes bietet dazu eine gelungene Erweiterung, um tiefer in die Thematik einzusteigen. Für alle diejenigen, die schon tiefer in der Thematik stecken, ist es durchaus gewinnbringend, einen generellen Abriss zum Nachhaltigkeitsthema zu lesen. Deutlich geworden ist ebenso, dass der integrative HGF-Ansatz unbedingt weiterentwickelt und dabei geschärft werden sollte: Es ist - aus meiner Sicht - einer der wenigen Nachhaltigkeitsansätze, der die integrative Perspektive ernst nimmt und der gleichzeitig durch die Verankerung in der HelmholtzGemeinschaft einen „langen Atem“ haben kann - im Gegensatz zu kurzfristigen FuE-Projekten von wenigen Jahren Dauer. 